\title{
Edad máxima en la que una mujer puede ser madre con sus propios óvulos. Revisión sistemática.
}

\author{
Gonzalo Acuña R. ${ }^{1}$, Juan Enrique Schwarze M., $M S c^{2,3}$, Sonia Villa V. ${ }^{3}$, \\ Ricardo Pommer T, 3,4 \\ ${ }^{1}$ Programa de Formación de Especialistas, Universidad de Santiago de Chile. ${ }^{2}$ Departamento Clínico de Obstetricia y \\ Ginecología, Universidad de Santiago. ${ }^{3}$ Unidad Medicina Reproductiva, Clínica Monteblanco. ${ }^{4}$ Instituto de Investigación \\ Materno Infantil de la Universidad de Chile.
}

\section{RESUMEN}

Antecedentes: La amplia realización de ciclos de ovodonación, en todo el mundo, se ha traducido en elevadas tasas de embarazos y partos en mujeres de diversas edades, incluyendo a postmenopáusicas tardías. Esto ha generado controversias y desafíos éticos, motivando a que muchos centros en los cuales se practican estas técnicas limiten la edad máxima de las mujeres receptoras. Objetivo: Buscar un criterio biológico y objetivo para definir esta edad, para conocer las edades máximas reportadas, en las cuales la mujer es capaz con sus propios gametos lograr un embarazo espontáneo y parto. Método: Se realizó una búsqueda amplia en las bases de datos de PUBMED y MEDLINE de artículos que reportaran mujeres que concibieron sobre 40 años y que especificaran la edad al momento del último parto, excluyéndose aquellos en los cuales se usaron técnicas de reproducción asistida. Resultados: Sólo dos trabajos completaron los criterios de inclusión y no presentaron los de exclusión. Las máximas edades reportadas fueron 49 y 52 años respectivamente. Conclusión: La edad máxima reportada en la literatura en la cual una mujer ha logrado un embarazo espontáneo y parto es a los 52 años.

\section{PALABRAS CLAVE: Envejecimiento de la mujer, fertilidad, embarazo espontáneo}

\section{SUMMARY}

Background: The broad use of oocyte donation cycles has resulted in high rates of pregnancy and child birth in women of different ages, including postmenopausal women. This has generated controversy and ethical challenges, motivating many centers to limit the maximum age of oocytes recipients. Aims: To seek a biological and objective criteria to define such maximal age, in which a woman is able to achieve a spontaneous pregnancy and give a birth. Methods: We performed a broad search PUBMED and MEDLINE including articles that reported women who conceived after age 40 years and specify the age at last birth, excluding those who received donated eggs. Results: Only two articles presented the inclusion criteria and no exclusion. The maximum reported ages were 49 and 52 years respectively. Conclusion: The maximum age reported in the literature in which a woman has achieved a spontaneous pregnancy and birth with her own gametes is at 52 years.

KEY WORDS: Female aging, fertility, spontaneous pregnancy 


\section{INTRODUCCIÓN}

Según progresa la edad de la mujer, su fecundidad experimenta una progresiva disminución. Es así como la fecundidad femenina se altera con la edad, mostrando una lenta y constante declinación entre los 20 y 35 años de edad, con una aceleración progresiva en esta disminución en los siguientes diez años, de modo que la frecuencia de embarazos y nacimientos de niños es rara después que la mujer cumple 45 años de edad (1).

Esta disminución de la fecundidad se atribuye a distintas causas, incluyendo cambios en los ovocitos, diminución de la frecuencia y eficiencia de la ovulación, disminución en la frecuencia coital, disminución de la receptividad uterina y complicaciones del embarazo (2-4).

Hasta el desarrollo de la tecnología de donación de gametos, era impensado que una mujer pudiera concebir y parir un bebé después de la menopausia. Sin embargo, en la actualidad no es raro el reporte de casos de mujeres en su quinta, o incluso sexta década, que paren un bebé producto de la donación de ovocitos de mujeres jóvenes $(5,6)$.

Dado que ya no existe esta limitación para conseguir embarazos, esto plantea la disyuntiva de hasta que edad realizar estos procedimientos. Si bien las mujeres que conciben naturalmente y tienen un parto después de los 45 años de edad representan un grupo selecto, excepcionalmente fértil, en el cual el envejecimiento ovárico esta diferido significativamente (1), y podrían servir como referencia para los médicos y pacientes que están considerando realizar un ciclo con donación de gametos.

El objetivo de este estudio es realizar una revisión sistemática de la literatura médica, para determinar hasta que edad se ha publicado que una mujer puede, espontáneamente y con sus propios ovocitos, concebir y tener un parto.

\section{MATERIAL Y MÉTODO}

Se buscaron artículos en la base de datos MEDLINE y PUBMED. Los criterios de búsqueda fueron amplios y correspondieron a la presencia en cualquier campo de las palabras claves "female aging", "fertility" y "spontaneous pregnancy". Luego se buscaron nuevos artículos dentro del listado de referencias de cada artículo. La cobertura temporal fue desde el año 1996 hasta marzo del 2013.

Los criterios de inclusión fueron publicaciones en inglés, castellano o alemán, que evaluaran mujeres que concibieron sobre 40 años y que especificaran la edad al momento del último parto. Los criterios de exclusión fue el uso de técnicas de reproducción asistida.

\section{RESULTADOS}

Luego de aplicar los criterios de búsqueda encontramos un total de 43 publicaciones, solo dos cumplieron con los criterios de inclusión y no presentaron los criterios de exclusión.

En 2004 Laufer y cols (7) publicaron un estudio en el cual caracterizaron la historia reproductiva de mujeres que tuvieron un parto después de un embarazo espontáneo después de cumplir 45 años de edad. Durante el periodo de estudio hubo un total de 104.659 partos, $209(0,2 \%)$ de los que correspondió a mujeres con al menos 45 años. La gravidez media fue $11,5 \pm 4,5$ embarazos (rango: $1-26$ ); $33 \% \mathrm{mu}$ jeres tuvieron $\geq 12$ embarazos, $16 \%$ tuvieron $\geq 16$ embarazos, y $1,5 \%$ tuvieron $\geq 22$ embarazos. La paridad promedio fue 9,6 \pm 4 niños (rango: $2-20$ ); $81 \%$ tenían $\geq 6$ partos; $46 \%$ tenían $\geq 11$ partos, y $2 \%$ tenían $\geq 18$ partos. La media de la edad del último parto fue 45,7 $\pm 0,9$ años (rango: 45-49 años).

En 2009, Gielchinsky y cols (8) publicaron un análisis retrospectivo de mujeres beduinas que concibieron espontáneamente después de los 45 años de edad entre los años 1995 y 2000. Durante el periodo de estudio hubo un total de 34.519 partos, $133(0,38 \%)$ de los cuales fueron en mujeres con 45 años o más. La paridad promedio fue 11,6 $\pm 2,9$ hijos (rango: 3-18 hijos). Estas mujeres tenían una alta tasa de multiparidad; $96 \%$ tenían $\geq 6$ partos; $70 \% \geq 11$ partos. La edad promedio del último parto fue $46 \pm 1,4$ años (rango: 45-52 años).

\section{DISCUSIÓN}

Al realizar la presente revisión intentamos responder la pregunta hasta que edad una mujer puede concebir y parir un bebé con sus propios óvulos, como una especie de control para médicos que realicen procedimientos de ovodonación. Ninguno de los estudios encontrados y revisados buscaban responder esta pregunta, sino que trataban el pronóstico de los embarazos en mujeres con 45 y más años, e incidentalmente informaban acerca de la máxima edad de las mujeres al momento del parto. En la seria publicada por Laufer y cols (7), la mujer de más edad tenia 49 años al momento del parto, y en la serie publicada por Gielchinsky y cols (8), tenía 52 años al momento del parto. De acuerdo al Registro Latinoamericano de Reproducción Asistida del 2010 (9), la mujer de más edad que tuvo un parto luego de un procedimiento de reproducción asistida con sus propios óvulos tenía 49 años al momento de realizarse el procedimiento.

Nuestra pregunta de investigación es especialmente pertinente en la actualidad, ya que muchas mujeres han retrasado su maternidad hasta la cuarta o incluso quinta década de la vida. Las razones 
para este retraso son múltiples e incluyen la prosecución de sus carreras profesionales y el matrimonio en forma tardía.

Asociado con el incremento de la edad las mujeres hay una clara disminución de la fecundidad, lo que puede ser obviado mediante la donación de ovocitos. Es así como la tasa de embarazo en mujeres receptoras de ovocitos donados es básicamente independiente de la edad de la receptora (9-11). Sin embargo, el aumento de la edad materna también se asocia con aumento de las tasas de enfermedad cardiovascular, diabetes, obesidad, leiomioma uterino y otras (12).

$\mathrm{Si}$ bien las series analizadas corresponden a la historia obstétrica de mujeres extremadamente fértiles, con un gran número de partos y gran multiparidad ( $\geq 6$ partos) $80 \%$ en el estudio de Laufer y cols (7) y $96 \%$ en el estudio de Gielchinsky y cols (8), consideramos que pueden servir de referencia y guía para los médicos que ofrezcan ciclos de donación de gametos.

\section{CONCLUSIÓN}

Encontramos que la mayor edad que se ha reportado una concepción espontánea y parto es en una mujer de 52 años, lo que puede servir de referencia para los centros de medicina reproductiva que ofrezcan donación de gametos.

\section{REFERENCIAS}

1. Klein J, Sauer MV. Assessing fertility in women of advanced reproductive age. Am J Obstet Gynecol 2001;185:758-70.

2. Rosen MP, Johnstone E, McCulloch CE, SchuhHuerta SM, Sternfeld B, Reijo-Pera RA, Cedars MI. A characterization of the relationship of ovarian reserve markers with age. Fertil Steril 2012;97:238-43.
3. Ramalho de Carvalho B, Gomes Sobrinho DB, Vieira $A D$, Resende MP, Barbosa AC, Silva AA, Nakagava HM. Ovarian reserve assessment for infertility investigation. ISRN Obstet Gynecol 2012;2012:576385. Disponible en: http://www.ncbi.nlm.nih.gov/pmc/articles/ PMC3302183/

4. Lambalk CB, van Disseldorp J, de Koning $\mathrm{CH}$, Broekmans FJ. Testing ovarian reserve to predict age at menopause. Maturitas 2009;63:280-91.

5. Tarlatzis BC, Zepiridis L. Perimenopausal conception. Ann NY Acad Sci 2003;99:793-104.

6. Ferraretti AP, Goossens V, de Mouzon J, Bhattacharya S, Castilla JA, Korsak V, et al. Assisted reproductive technology in Europe, 2008: results generated from European registers by ESHRE. Hum Reprod 2012;27:2571-84.

7. Laufer N, Simon A, Samueloff A, Yaffe H, Milwidsky A, Gielchinsky Y. Successful spontaneous pregnancies in women older than 45 years. Fertil Steril 2004;81:1328-32.

8. Gielchinsky Y, Mazor M, Simon A, Mor-Yossef S, Laufer N. Natural conception after age 45 in Bedouin women, a uniquely fertile population. J Assist Reprod Genet 2006;23:305-9.

9. Zegers-Hochschild F, Schwarze JE, Galdames V. XIX Registro Latinoamericano de Reproducción Asistida 2008. 2010. Disponible en: http://www.redlara.com/ images/arq/2008_registro\%202008.pdf.

10. Bodri D, Colodron M, Vidal R, Galindo A, Durban $\mathrm{M}$, Coll O. Prognostic factors in oocyte donation: an analysis through egg-sharing recipient pairs showing a discordant outcome. Fertil Steril 2007;88:1548-53.

11. Oyesanya OA, Olufowobi O, Ross W, Sharif K, Afnan M. Prognosis of oocyte donation cycles: a prospective comparison of the in vitro fertilization-embryo transfer cycles of recipients who used shared oocytes versus those who used altruistic donors. Fertil Steril 2009,;92:930-6.

12. Callaway LK, Lust K, McIntyre HD. Pregnancy outcomes in women of very advanced maternal age. Aust $N$ Z J Obstet Gynaecol 2005;45:12-6. 